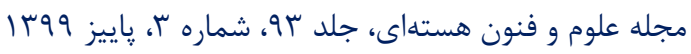

Journal of Nuclear Science and Technology

Vol. 93, No. 3, 2020

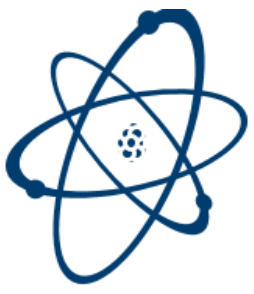

غنىسازى ايزوتوڤ Zr?" به روش جداسازى الكترومغناطيسى ايزوتوبها

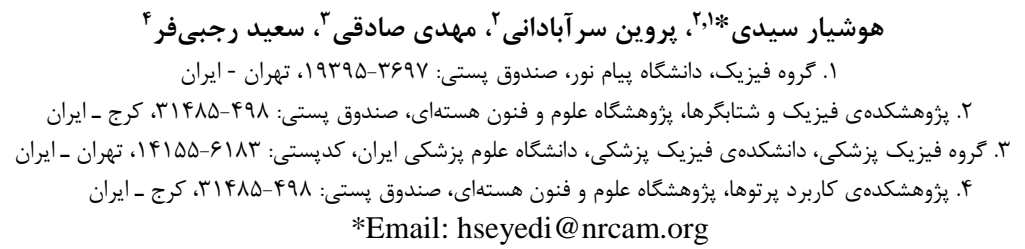

مقالهى : ثزوهشى

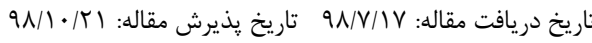

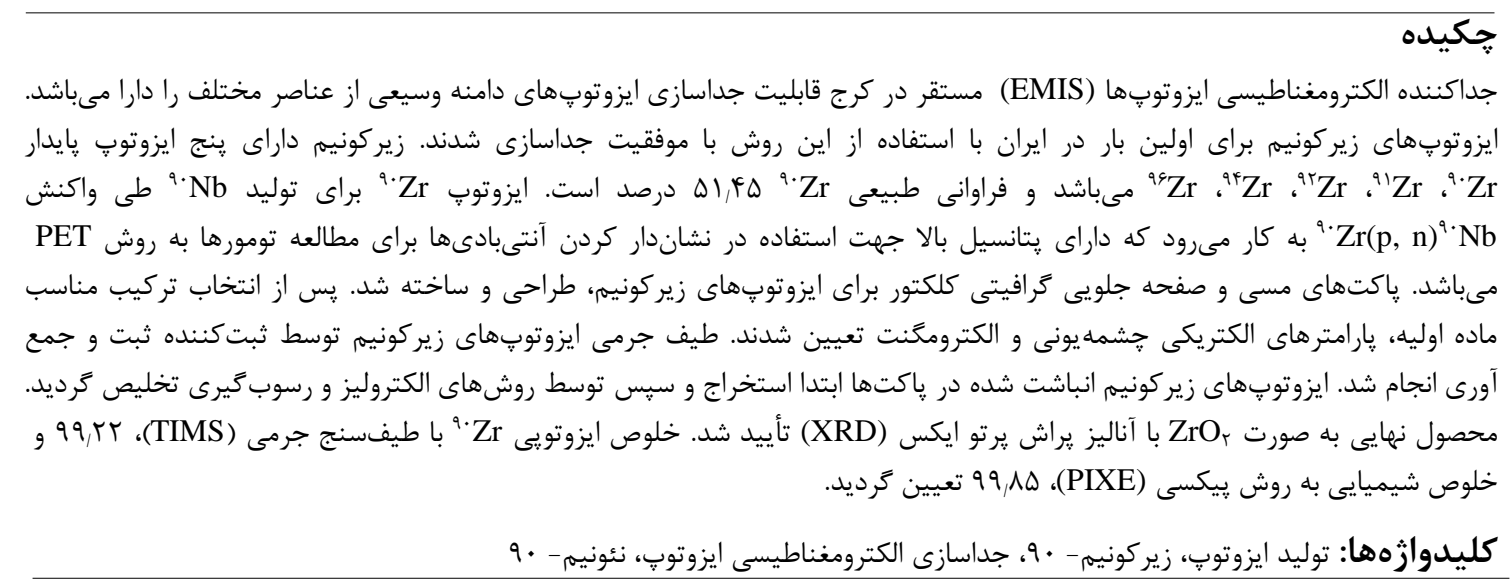

\title{
Enrichment of ${ }^{90} \mathrm{Zr}$ by Electromagnetic Isotope sSeparation (EMIS)
}

H. Seyedi*1,2, P. Sarabadani², M. Sadeghi ${ }^{3}$, S. Rajabifar $^{4}$

1. Department of Physics, Payame Noor University, P.O.Box: 19395-3697, Tehran - Iran

2. Physics and Accelerator Research School, Nuclear Sciences and Technology Research Institute, P.O.Box: 31485-498, Karaj - Iran 3. Medical Physics Department, School of Medicine, Iran University of Medical Sciences, Postalcode: 14155-6183, Tehran - Iran

4. Radiation Application Research School, Nuclear Sciences and Technology Research Institute, P.O.Box: 31485-498, Karaj -Iran

Research Article

Received 9.10.2019, Accepted 11.1.2020

\begin{abstract}
The Electromagnetic Isotopes Separator (EMIS) installed in Karaj, has the capability to separate isotopes in a wide range of elements. Zirconium is successfully separated using this method for the first time in Iran. Zirconium element has five stable isotopes, ${ }^{90} \mathrm{Zr}$, ${ }^{91} \mathrm{Zr},{ }^{92} \mathrm{Zr},{ }^{94} \mathrm{Zr}$, and ${ }^{96} \mathrm{Zr}$, and the natural abundance of ${ }^{90} \mathrm{Zr}$ is $51.45 \%$. The ${ }^{90} \mathrm{Zr}$ isotope is used to produce radioisotope ${ }^{90} \mathrm{Nb}$ via ${ }^{90} \mathrm{Zr}(\mathrm{p}, \mathrm{n}){ }^{90} \mathrm{Nb}$ that has a high potential for antibody labeling application in PET as a radionuclide. Copper pockets and graphite front plate were designed and fabricated for separating and collecting of $\mathrm{Zr}$ isotopes. After choosing the appropriate composition for the initial material, the electrical parameters of the ion source and electromagnet were determined. The mass spectra of Zirconium isotopes were recorded. Deposited ${ }^{90} \mathrm{Zr}$ isotopes were first extracted from the copper pocket and then purified by electrodeposition and purification methods. The formation of ${ }^{90} \mathrm{ZrO}_{2}$ was confirmed by X-ray diffraction (XRD). Chemical purity and isotope purification of ${ }^{90} \mathrm{Zr}$ isotope were $99.22 \%$ and $99.85 \%$, which measured by TIMS and PIXE method analysis, respectively.
\end{abstract}

Keywords: Isotope Production, Zr-90, Electromagnetic Isotope Separation, Nb-90

Journal of Nuclear Science and Technology

مجله علوم و فنون هستهاي Vol. 93, No 3, 2020, P 81-86

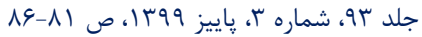




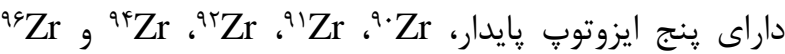

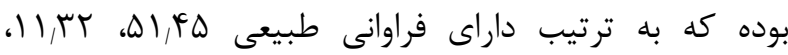
IVI/19

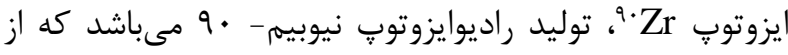

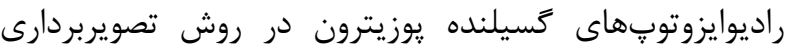

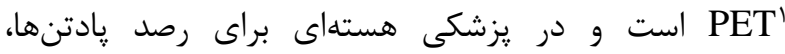
تشخيص و مطالعات زيستى تومور، توان بالقوه بالايى دارد.

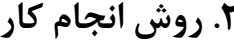

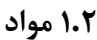

كلريد زيركونيم به صورت \& شركت MERCK، اسيد مندليك، اسيد نيتريك، اسيد كلريدريك و هيدرواكسيدآمونيم از شركت MERCK تهيد

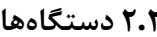

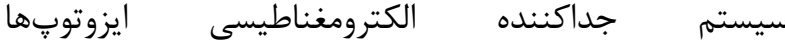
مدل .CI-IY، دستخاه يراش اشعه ايكس (مدل ،W-Ka X ray source Stol Stidy-Mp diffractometer) دستخاه شتابدهنده الكترواستاتيكى واندوكراف با باريكه يروتون PIXE) rMeV)، دستغاه طيفسنج جرمى مدل

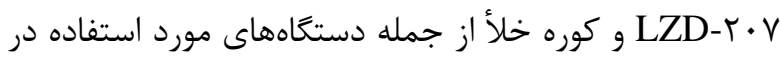

$$
\text { اين كار يزوهشى بودند. }
$$

\section{Y.r ا بتخاب و آمادهسازى ماده اوليه}

ماده اوليه يك تركيب شيميايى از عنصر مورد نظر است كه

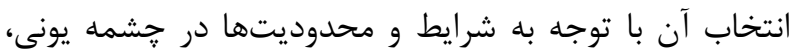

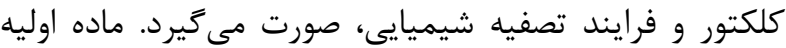

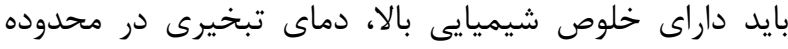

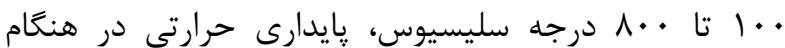

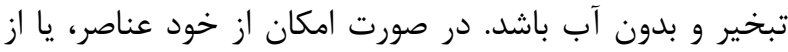
كلريد و اكسيد عناصر مىتوان استفاده نمود. براى غنى إنىسازى إنى

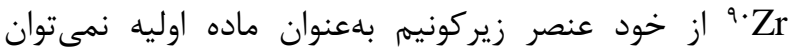

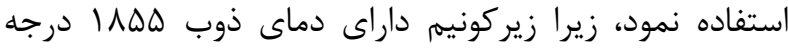

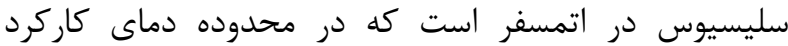

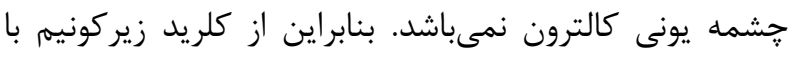

مشخصات مندرج در جدول [استفاده شد [9].

\begin{tabular}{|c|c|c|c|}
\hline فرمول مولكولى & 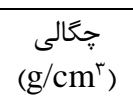 & $\begin{array}{c}\text { دماى ذوب } \\
\left({ }^{\circ} \mathrm{C}\right)\end{array}$ & $\begin{array}{c}\text { دماى تبخير } \\
\left.{ }^{\circ} \mathrm{C}\right)\end{array}$ \\
\hline$\overline{\mathrm{ZrCl}_{f}}$ & $r, \wedge$. & Fry & Trו \\
\hline
\end{tabular}

1. Positron Emission Tomography

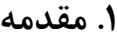

روشهاى مختلفى مانند روش سانتريفوز گازى، جداسازى ايزوتويى ليزرى، جداسازى به روش تشديد سيكلوترونى يونها

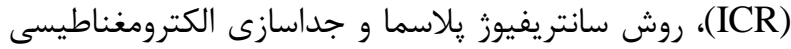

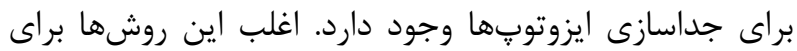

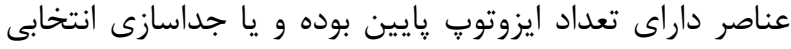

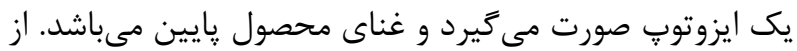

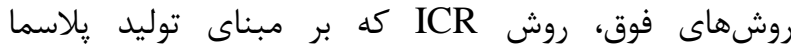
سيكلوترونى پايهريزى شده است، اكنون بهاعنوان يكى روش

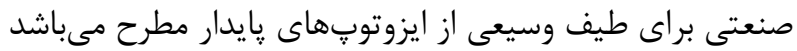

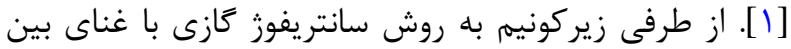

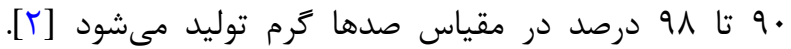

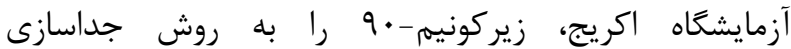

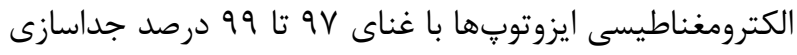

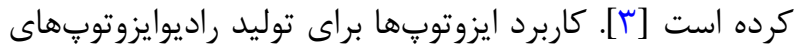

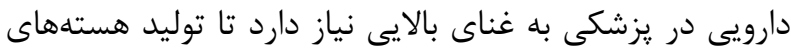

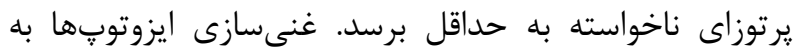

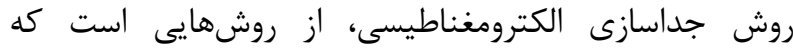

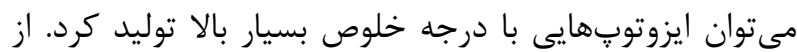

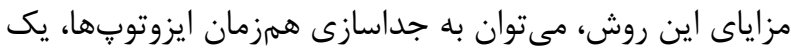

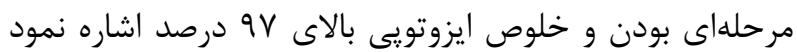

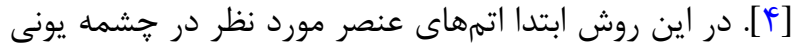

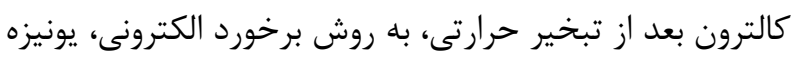

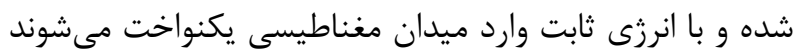

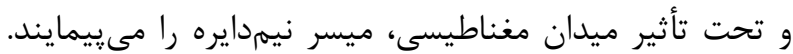

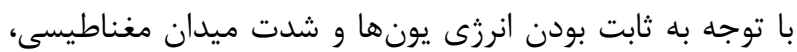

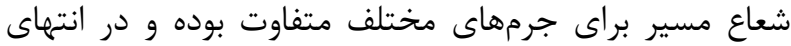

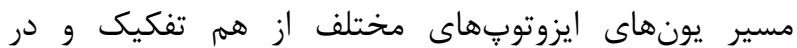

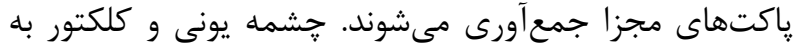

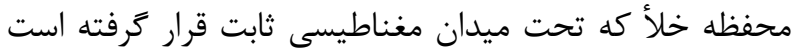

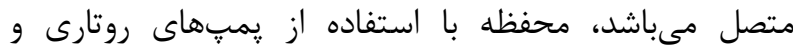

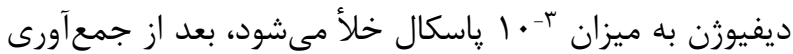

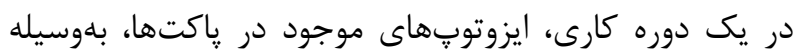

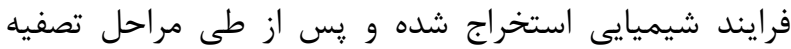

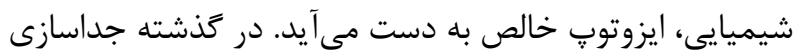

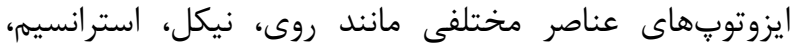

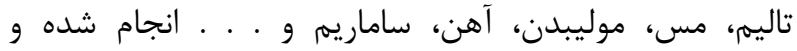
محصولات آنها در مراكز مربوط به توليد النيد راديوداروها و صنايع

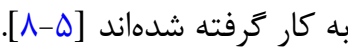

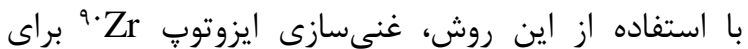
نخستين بار در ايران با موفقيت انجام شد. عنصر زيركونيم إنيم 


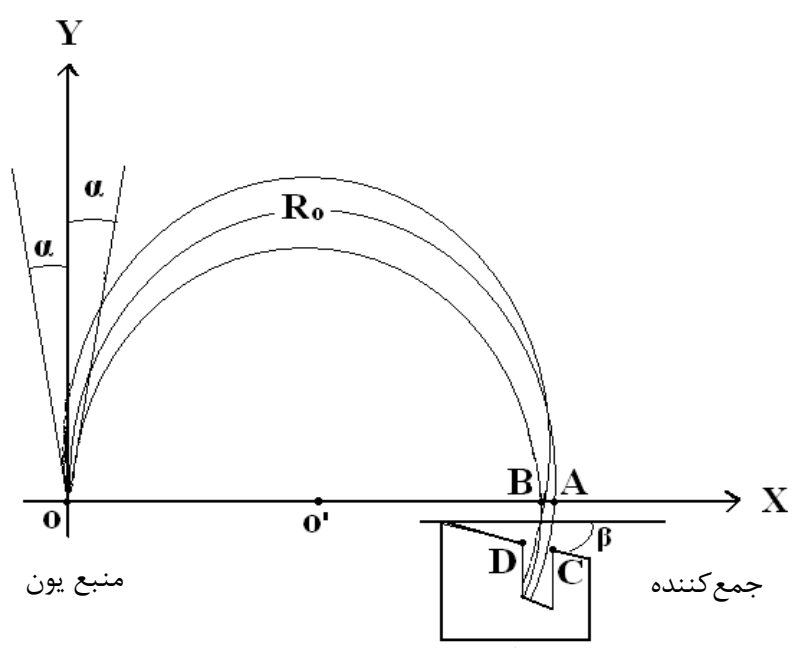

شكل ا. مسير طى شده يك ايزوتوٍ در ميدان مغناطيسى.

جدول r. مختصات پاكتهاى ايزوتوٍ هاى زيركونيم در كلكتور

\begin{tabular}{|c|c|c|c|c|c|}
\hline${ }^{98} \mathrm{Zr}$ & ${ }^{94} \mathrm{Zr}$ & ${ }^{9 r} \mathrm{Zr}$ & ${ }^{9} \mathrm{Zr}$ & ${ }^{9} \cdot \mathrm{Zr}$ & زيروتوبهاى \\
\hline T,VG & $I V, r \Lambda$ & 18,19 & $11, \pi r$ & $\Delta 1,4 \Delta$ & درصد فراوانى \\
\hline $1, \cdot r r$ & $1, \cdot 11$ & .9419 &., $9 \vee \wedge$ & $.99 \mathrm{~V}$ & مقدار CM \\
\hline$r \wedge \varphi 1, \Lambda$ & TATY, & $r \Lambda \cdot \Delta$ & TVAF & $T V S V / D$ & مقدار X(mm) \\
\hline$-199,1$ & $-1 \Gamma \Delta, \Delta$ & $-1 \cdot \cdot \Delta$ & $-\lambda r, r$ & $-99,1$ & مقدار Y(mm) \\
\hline
\end{tabular}

نقشه صفحه جلويى و پاكتها در شكل ب آورده شده است.

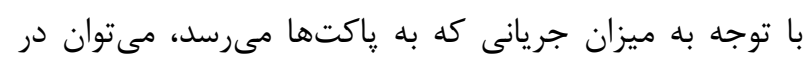

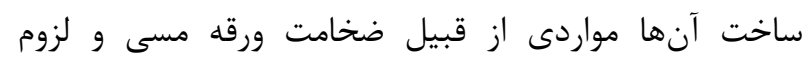

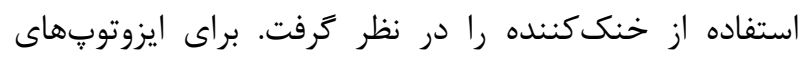

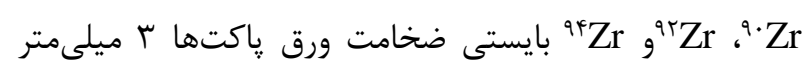

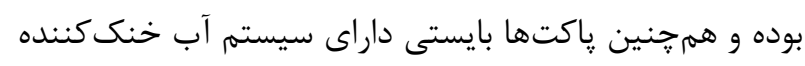

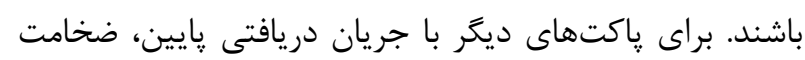

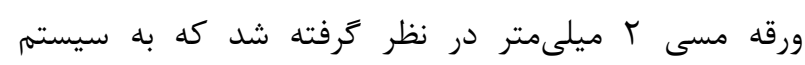

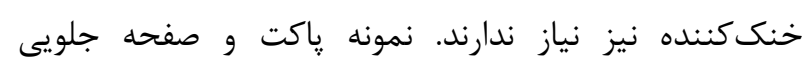
كرافيتى ساخته شده در شكل ب نشان داده شده است. ندارن

\section{D. به دست آوردن شرايط كار}

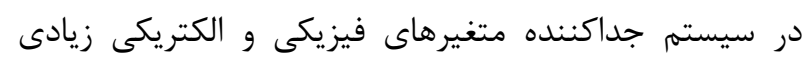

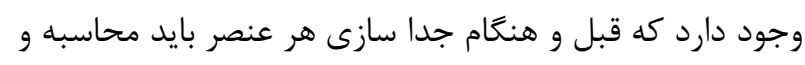

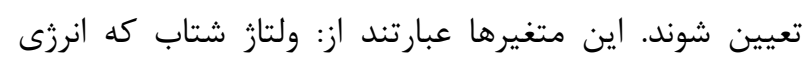

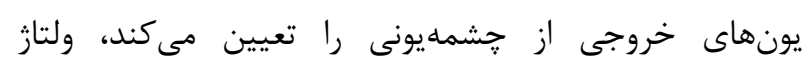

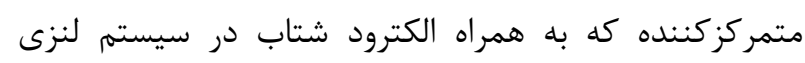

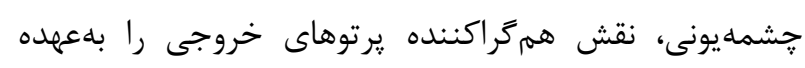

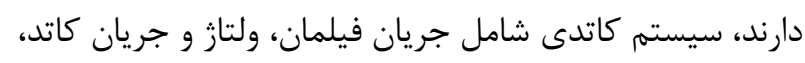
ولتاز آند كه شدت و انرزى الكترونهاى ساطع شده راندي ران كنترل

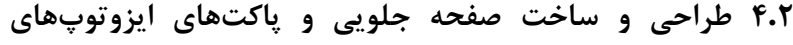
زير كونيم با توجه به شكل ا، مسير يرتوها در ميدان مغناطيسى

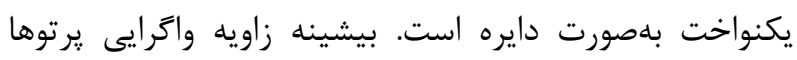
براى هر ايزوتوٍ در خشمه يونى لم فرض شده است. بنابراين

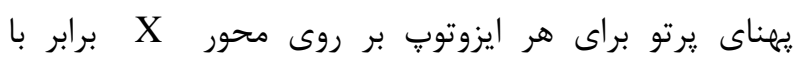

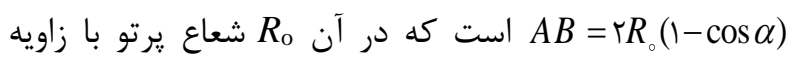
واكرايى صفر مىباشد. اين بهنا در روى كلكتور كه با محور

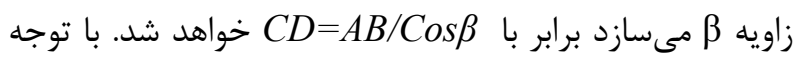

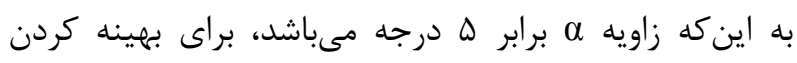

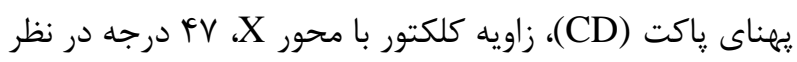

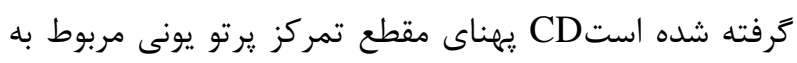

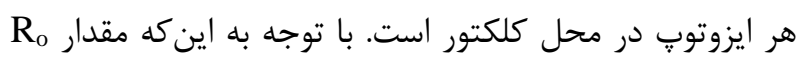

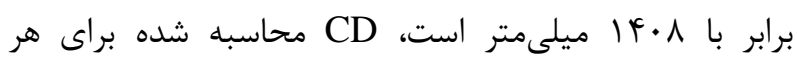

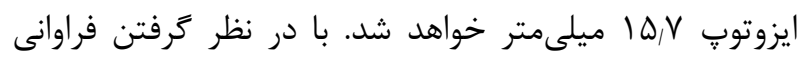

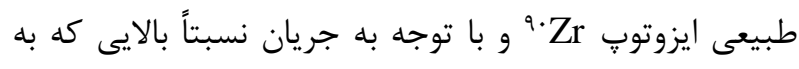

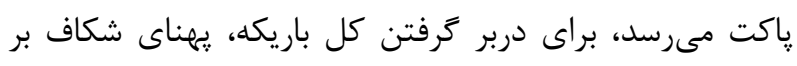

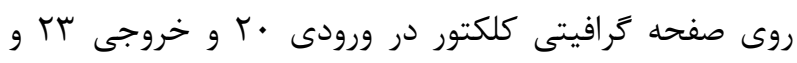

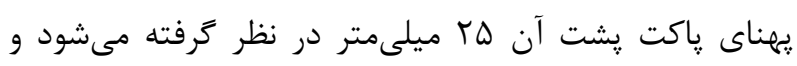

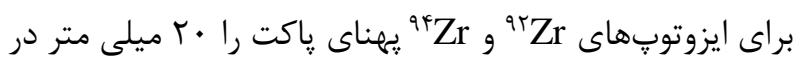
نظر كرفته شد.

مختصات مركز هر ياكت كه از قطع دماند دادن معادلات دايرههاى مربوطه و خط كلكتور محاسبه شده و و هارامتر نسبت جرم هر ايزوتوب به جرم ايزوتوٍ ميانى (C $\left.C_{M}=\frac{M}{M}\right)$

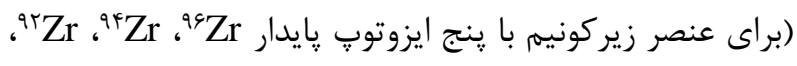

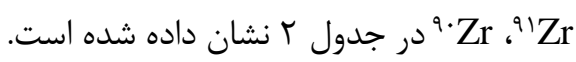

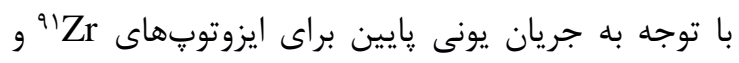

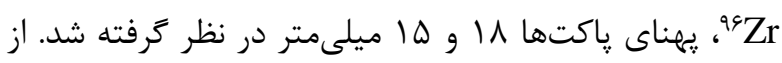

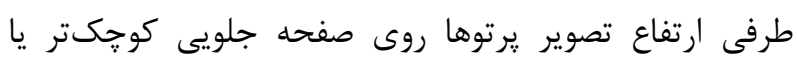

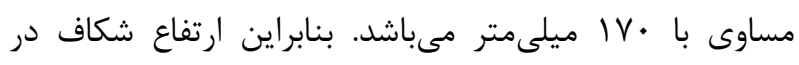

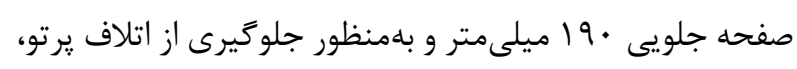

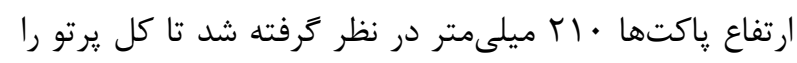

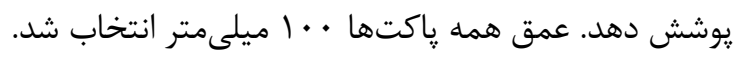


مقدار شدت ميدان مغناطيسى حدود IVIF كوس محاسبه شد كه لازم است با توجه به مشخصات مخَت جريان سيمييج

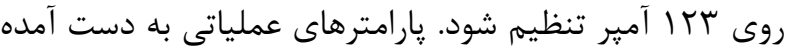

در جدول ب آمده است.

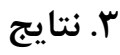

ץ.ا جداسازى ايزوتوضهاى زيركونيم

بعد از تنظيم يارامترهاى ذكر شده و رسيدن به به شرون ايط مطلوب

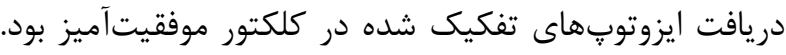

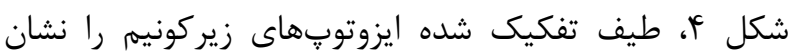
مىدهد كه توسط يك فنجان فارادى موجود روى درب كلكتور ثبت شده است. در اين فرايند، با تغيير ولتاز شتابدهنده و ورئ

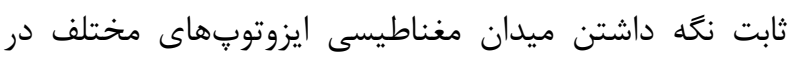

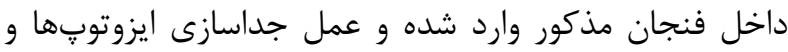

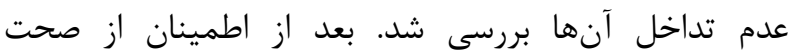

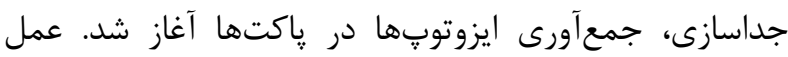
جمعآورى حدود هفتاد و جهار ساعت با ميانگين جريان كل r r mA

\section{r.r تصفيه شيميايى ايزوتوب Zr Ir و آناليزها}

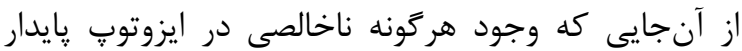
زيركونيه- • 9 منجر به ايجاد واكنشهاى ناخواسته هستهاى در

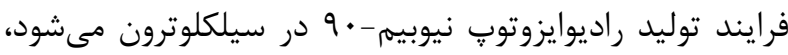
لذا تصفيه شيميايى ايزوتوب يايدار زيركونيم-•9 از اهميت

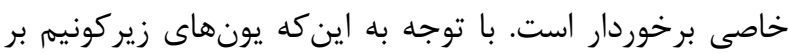

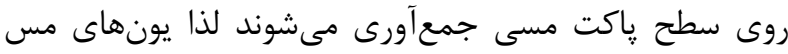

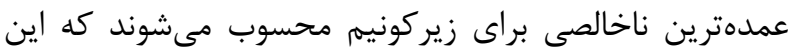

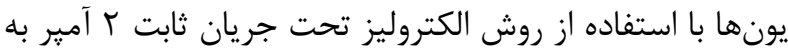

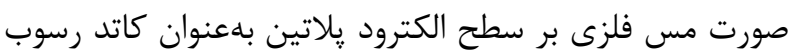

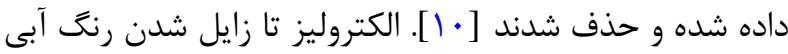
مربوط به يون مس ادامه يافت. با توجه به وجود ناخالصىهاى

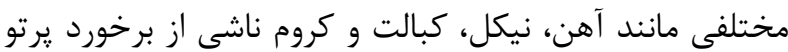

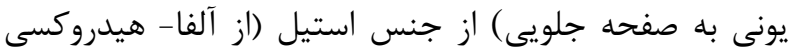
فنيل استيك اسيد با نام تجارى مندليك اسيد بهوعنوان

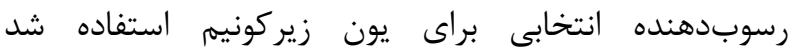

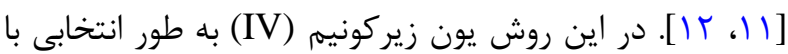

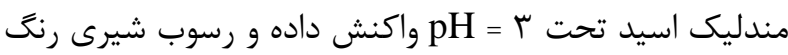

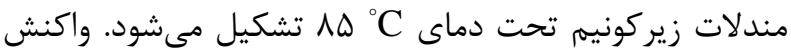
تشكيل كميلكس زيركونيم (IV) با مندليك اسيد به صوريه صورت

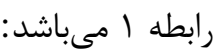

$$
\mathrm{Zr}^{\mathrm{r}_{+}}+{ }_{\mathrm{r}} \mathrm{C}_{\lambda} \mathrm{H}_{\lambda} \mathrm{O}_{r} \rightarrow \mathrm{Zr}\left(\mathrm{C}_{\Lambda} \mathrm{H}_{\Lambda} \mathrm{O}_{r}\right)^{\mathrm{r}}
$$

مى كنند، فشار بخار در محفظه قوس الكتريكى كه نقش تعيين كننده در ايجاد يلاسما داشته و توسط هيترهاى مربوطه

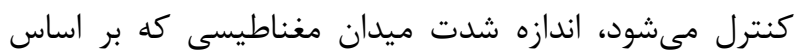
محاسبات تئورى، منحنى و جداول سيستم [ه] با توجه به ميه ثابت بودن ولتاز شتاب، مقدار آن براى جرم ايزوتوتٍ ميانى از

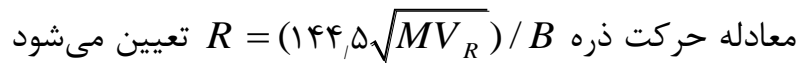
كه R، شعاع مسير يون برحسب سانتىمتر، B، ميدان مغناطيسى برحسب گوس، ${ }^{\prime}$ ولتاز شتابدهنده (برحسب ولت) و M عدد جرمى يون ها مىباشند.

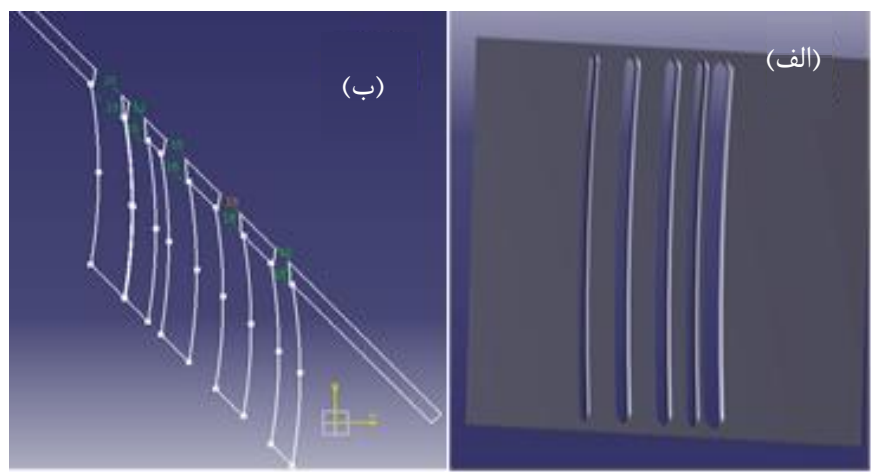

شكل r. الف) صفحه گرافيتى جلويى با شكافهاى ينجزانه براى ينج

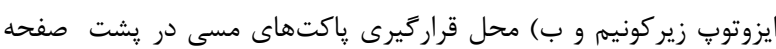
كرافيتى جلويى در كلكتور.

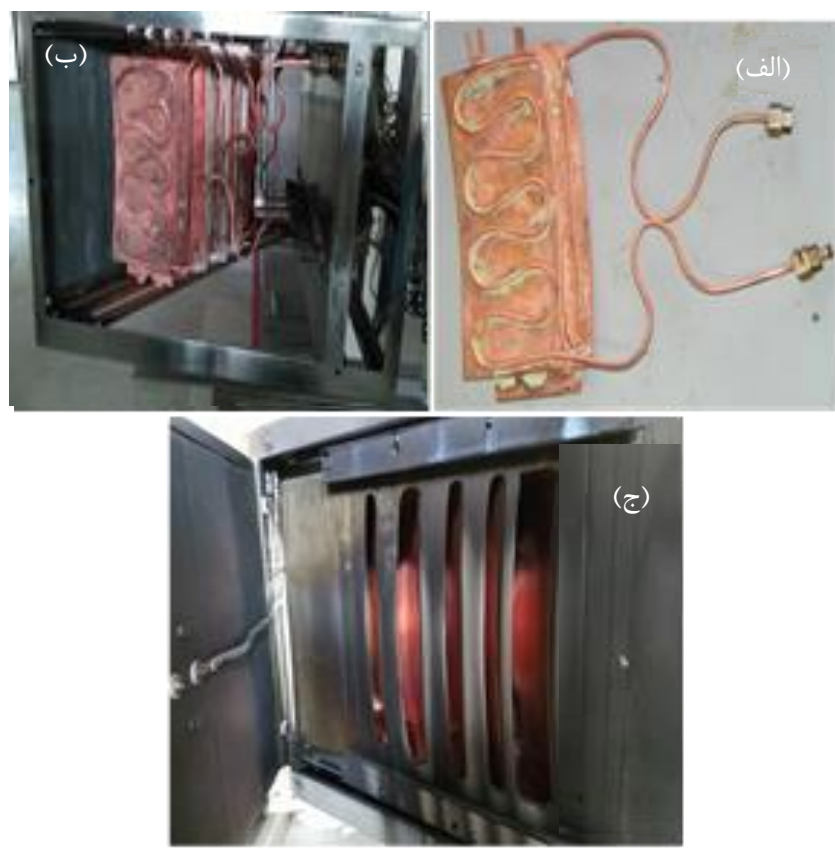

شكل س. الف) نمونه پِاكتهاى مسى ساخته شده، ب و ج) نحوه قراركيرى

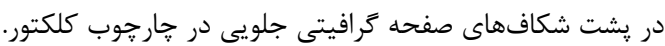


جدول س. متغيرهاى عملياتى در هنعام جداسازى ايزوتوبهاى زيركونيم

\begin{tabular}{|c|c|c|c|c|c|c|c|c|c|c|}
\hline $\begin{array}{l}V_{\text {Acc }} \\
(\mathrm{kV}) \\
\end{array}$ & $\begin{array}{l}V_{\text {Foc }} \\
(\mathrm{kV})\end{array}$ & $\begin{array}{l}\mathrm{I}_{\mathrm{Fi}} \\
(\mathrm{A})\end{array}$ & $\begin{array}{l}\mathrm{V}_{\text {Cat }} \\
(\mathrm{V})\end{array}$ & $\begin{array}{l}\mathrm{I}_{\mathrm{Cat}} \\
(\mathrm{A})\end{array}$ & $\begin{array}{c}\mathrm{V}_{\text {Anode }} \\
(\mathrm{V})\end{array}$ & $\begin{array}{c}\mathrm{I}_{\text {Anode }} \\
\text { (A) }\end{array}$ & $\begin{array}{l}\mathrm{I}_{\text {Mag. }} \\
\text { (A) }\end{array}$ & $\begin{array}{l}\text { Vacuum } \\
(\mathrm{Pa})\end{array}$ & $\begin{array}{c}\text { Temp } \\
\left({ }^{\circ} \mathrm{C}\right)\end{array}$ & $\begin{array}{l}\mathrm{I}_{\text {Total }} \\
(\mathrm{mA}) \\
\end{array}$ \\
\hline$r \cdot \Lambda$ & $1 \cdot r$ & r & $v_{*}$ & $\cdot, i \wedge$ & 194 & - & rזו & $r \times 1 \cdot{ }^{-r}$ & Ira & 11 \\
\hline
\end{tabular}

\section{f. نتيجه}

در اين بزوهش، براى اولين بار در ايران ايزوتوٍِهاى زيركونيم

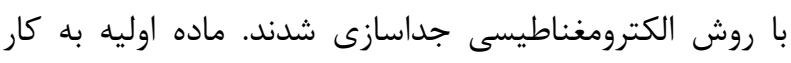

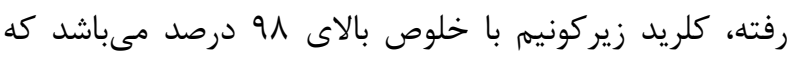

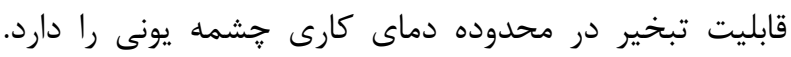

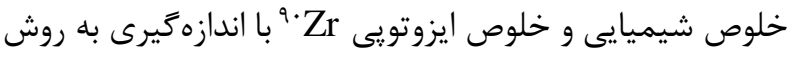

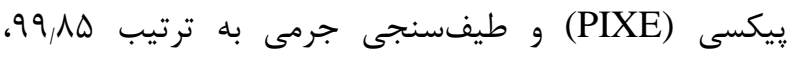

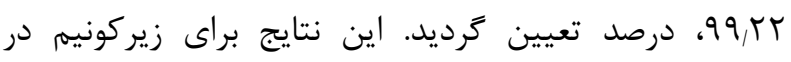

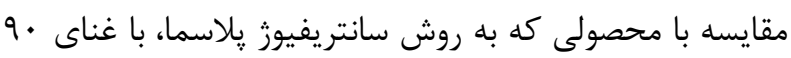

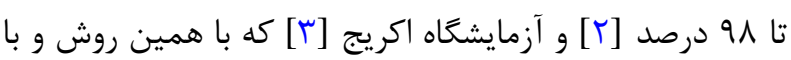

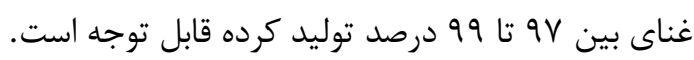
جدول F. نتايج مربوط به اندازميرى خلوص شيميايى به روش (PIXE)

\begin{tabular}{|c|c|c|c|c|c|}
\hline $\mathrm{CaO}$ & $\mathrm{Fe}_{\varphi} \mathrm{O}_{r}$ & $\mathrm{Cu}_{\uparrow} \mathrm{O}$ & $\mathrm{ZnO}$ & $\mathrm{ZrO}_{r}$ & تركيبات \\
\hline$\cdot 1 \cdot$ & $\cdot \cdot \Delta$ & - & - & $१ ৭, \wedge \Delta$ & درصد \\
\hline
\end{tabular}

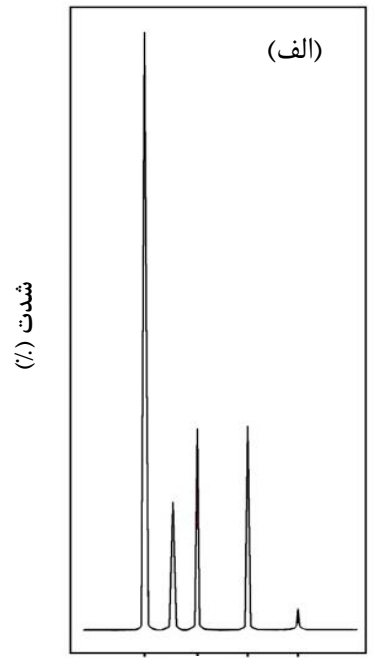

q. 94 qf 98 $\mathbf{m} / \mathbf{e}$

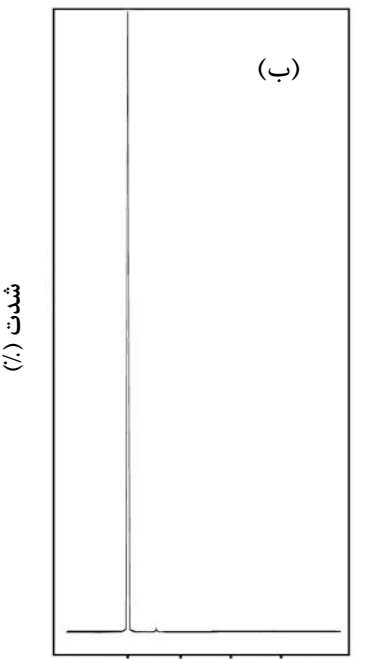

q. or qr $q 4$ $\mathbf{m} / \mathbf{e}$
شكل ه. طيف به دست آمده از طيفسنجى جرمى، الف) زيركونيم طبيعى غنى

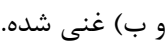

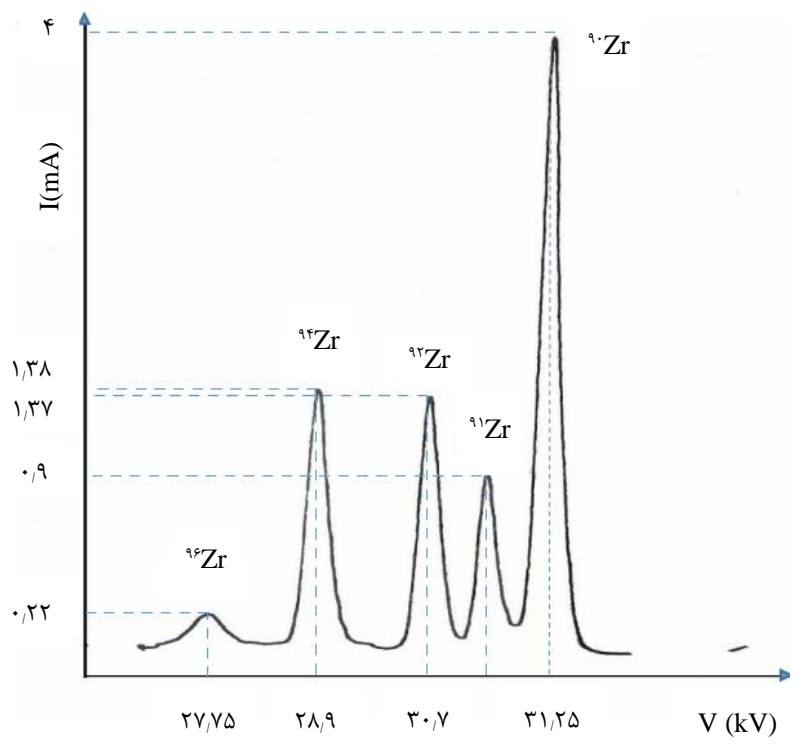

شكل f. طيف ثبت شده توسط ثبتكننده به هنكام جاروب ولتاز

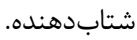

با توجه به اينكه محصول نهايى بايستى داراى تركيب و

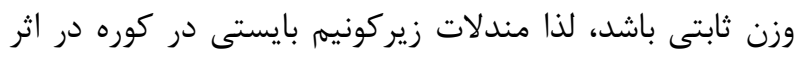

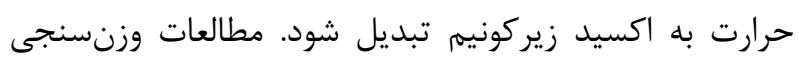

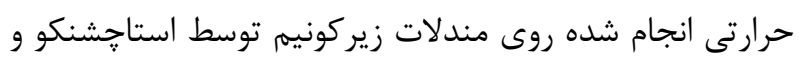

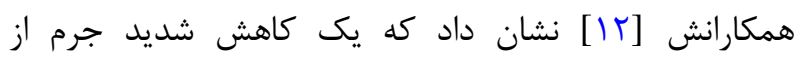

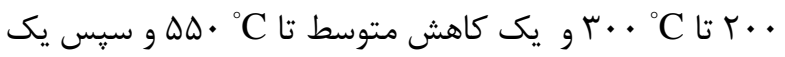

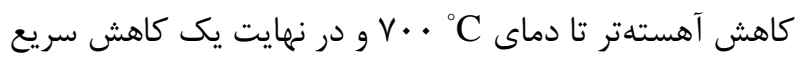

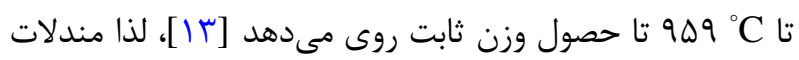

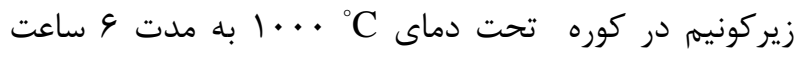

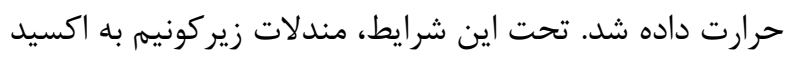

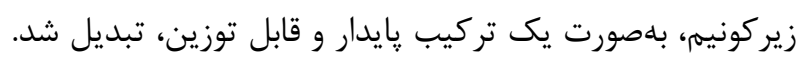

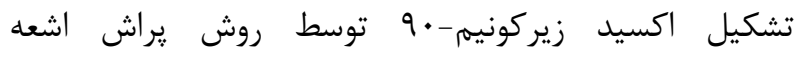

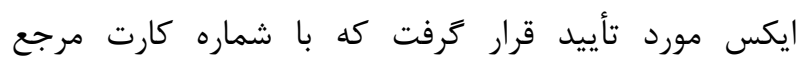

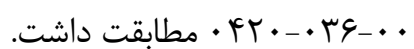

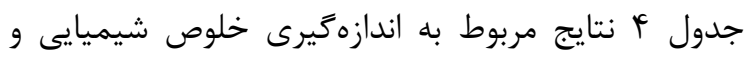

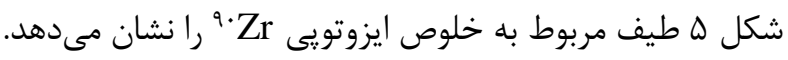


1. J.M. Dawson, Isotope separation in plasmas by use of Ion Cyclotron Resonance, Phys. Rev. Lett. 37(23), 1547 (1976).

2. H. Schmeing, W.J.L. Buyers, G. Dolling, Isotope Enrichment in Zirconium with a Plasma Centrifuge, Final report on contract CR31-3946, Chalk River Nuclear Laboratories, Atomic Energy of Canada, (June 1983).

3. W.A. Bell, J.G. Tracy, Stable Isotope inventory requirements and enrichment capabilities, Oak Ridge National Laboratory, USA, (December 1985).

4. J. Koch, Electromagnetic Isotope Separators and Application of Electromagnetically Enriched Isotopes, (Interscience Publishers, New York, 1958).

5. A.J. Novinrooz, et al., Separation of Thallium Isotope $\left.{ }^{203} \mathrm{Tl}\right)$ by $180^{\circ}$ Electromagnetic Isotope Separator, J. Nucl. Sci. Tech. AEOI, 28 (1382) (In Persian).

6. J. Garousi, et al., Recovery of Mo-98 Enriched Stable Isotope from Graphite Collector of EMIS and Chemical Purification, J. Nucl. Sci. Tech. AEOI, 37 (1385) (In Persian).

7. Z. Asadollahi, et al., Enrichment of $\mathrm{Fe}-54$ by Electromagnetic Isotope Separator (EMIS), J. Nucle. Sci. Tech., AEOI, 66 (1392) (In Persian).
8. S.M. Mohati, Enrichment of ${ }^{58} \mathrm{Ni}$ by electromagnetic isotope separator, $5^{\text {th }}$ International conference on isotopes, Brussels (Belgium), April 25-29 (2005).

9. C.W. Sheridan, H.R. Gwinn, L.O. Love, Preparation of Charge Materials for ORNL Electromagnetic Isotopes separator, ORNL- 3301 (Aug 8, 1962).

10. P. Sarabadani, et al., Chemical recovery and purification of ${ }^{203} \mathrm{Tl}$ stable isotope enriched by using an electromagnetic isotope separator, Journal of Labeled compounds and Radiopharmaceuticals, 50, 5-6 (2007).

11. B. Weaver, Chemical refinement procedure in the electromagnetic separation of isotopes, Oak Ridge National Laboratory, W-7405-eng-26, (1955).

12. G. Norwitz, Determination of Zirconium in Zirconium Metal and Zirconium Powder by Use of Mandelic Acid, Analytica Chimica Acta, 35, 491498 (1966).

13. J. Stachtchenko, C. Duval, Sur la thermogravimétrie des précipités analytiques Dosage du zirconium, Analytica Chimica Acta, 5, 410-421 (1951).

\section{COPYRIGHTS}

C2021 The author(s). This is an open access article distributed under the terms of the Creative Commons Attribution (CC BY 4.0), which permits unrestricted use, distribution, and reproduction in any medium,

as long as the original authors and source are cited. No permission is required from the authors or the publishers.

$$
\begin{aligned}
& \text { استناد به اين مقاله }
\end{aligned}
$$

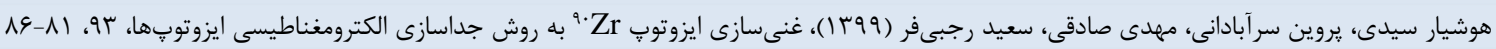

DOI: $10.24200 /$ nst.2020.1146

Url: https://jonsat.nstri.ir/article_1146.html 